\title{
Optimal Placement of TCSC and SVC Using PSO
}

\author{
K.Kavitha ${ }^{\# 1}$, Dr.R.Neela ${ }^{* 2}$ \\ \# Assistant Professor,Department of Electrical engineering, Annamalai University Chidambaram, Tamil nadu, \\ India. \\ * Professor,Department of Electrical engineering, Annamalai University Chidambaram, Tamil nadu, India.
}

\begin{abstract}
Flexible AC Transmission System (FACTS) devices play a vital role in improving the static as well as dynamic performance of the power system. They serve to make the otherwise rigid, conventional AC systems flexible ones as they make it possible to adjust the bus voltage magnitudes, phase angles and line impedances at faster rates in the required directions which will result in the improvement of stability and bus voltage profiles and reduction of real and reactive power losses. In order to tap maximum benefits out of their installation, the location and ratings of the FACTS devices are to be chosen optimally. In this paper a PSO based technique has been presented for solving the problem of device placement and an objective function comprising the weighted sum of the indicators of line loadings, bus voltage deviations and device cost functions has been formulated. The objective function is solved through the application of PSO technique and testing has been done using IEEE 14,30 and 57 bus systems
\end{abstract}

Keywords: FACTS devices, Thyristor Controlled Series Capacitor (TCSC), Static Var Compensator (SVC), Particle Swarm Optimization (PSO), Security enhancement.

\section{INTRODUCTION}

Present day power systems are highly complex interconnected systems, so it becomes necessary to operate the power systems in a secure and reliable manner. Due to the dynamic load pattern and ever increasing load demand, power flows in some of the transmission lines are well above their normal limits while some of the lines are not loaded upto their full capacity. As a result of this uneven load distribution, the voltage profile of the system gets deteriorated which poses a threat for the security of the system. Taking into consideration the factors such as ever increasing load demand, economical and technical constraints involved in setting up new power generation facilities and limitations faced in purchasing right of ways to realize new transmission corridors it becomes highly essential to utilize the existing generation and transmission facilities in the most efficient manner. FACTS controllers are found to be an effective alternative for the complex task of building up new transmission corridors [1].

Modulating and reversing the power flow through the transmission lines in a fast, accurate and precise manner was made possible through the FACTS (Flexible Alternating Current Transmission System) concept introduced by N.G.Hingorani [2]. FACTS devices are very effective in improving the voltage profile, reducing the line loadings and line losses, providing reactive power support over a wide range of operating voltages and enhancing the stability of the system. They can as well be used with the existing lines in order to enhance their power transfer capability. The power flow through the network can be controlled without modifying the generation and carrying out any switching operations in the network. [3]. In order to achieve maximum benefits through the installation of the FACTS devices, devices of suitable ratings need to be installed at optimal locations [4].

The proposed work deals with the placement of multi-type FACTS devices namely SVC and TCSC. SVC (Static Var Compensator) was the first device to be released in the market, when the concept of generating controllable reactive power through switching power converters was introduced. It is a shunt connected device and is installed parallel with a bus. It has the ability to generate or absorb reactive power at the point where it is connected. More than 800 SVC's are being installed worldwide both for utility and industrial purposes. (especially in electric arc furnace and rolling mills)[5].

TCSC (Thyristor Controlled Series Capacitor) is a type of series compensator that can provide many benefits for a power system including control of power flow in the line, damping power oscillations and mitigating sub synchronous resonance [6].TCSC is a variable impedance type series compensator. It consists of a series compensating capacitor shunted by a thyristor controlled reactor. By controlling the firing angle of thyristor, TCSC can change the line reactance smoothly and rapidly. TCSC has one of the two possible characteristics either capacitive or inductive, can be used for increasing or decreasing the reactance of the line $X_{l}[7]$. Moreover to avoid the over compensation of the line, the maximum values of capacitance and inductance are fixed at $-0.8 X_{l}$ and $0.2 X_{l}$ [8]. World's first 3 phase, $2 * 165$ MVAR, TCSC was installed in 1992 in Kayenta substation, Arizona. It raised the transmission capacity of transmission line by $30 \%$ and effectively damped electromechanical power oscillations [9]. Optimal placement of FACTS devices is essential to tap the maximum benefits in terms of system performance and cost effectiveness.

A loss sensitivity index with respect to the control parameters of FACTS devices has been suggested and with the computed loss sensitivity index, the FACTS devices are placed on the most sensitive bus or line [10]. Fuzzy based approach for the optimal placement of FACTS device for enhancing the system security under normal and network contingencies has been discussed in [11]. The optimal location of a given number of FACTS devices is a problem of combinatorial analysis. 
To solve such kind of problems, heuristic methods can be used [12]. They permit to obtain acceptable solutions within a limited computation time. The application of Genetic Algorithm for the optimal location of multi type FACTS devices in order to maximize the system loadability is analysed in [13]. A Differential Evolution based algorithm to decide the optimal location and device rating has been suggested in [14] with an objective of enhancing the system security under single line contingencies. The Particle Swarm Optimization (PSO) is applied for the optimal location of FACTS devices to achieve minimum cost of installation and to improve system loadability, by considering thermal limit for the lines and bus voltage limit for the load buses as constraints [15]. Sensitivity analysis approach for finding the optimal location and PSO for the optimal parameter setting of TCSC has been suggested in [16] so as to maximize the loadability.

In this paper, PSO technique is applied to find the optimal placement and capacity of multi - type FACTS devices (TCSC \& SVC). The objective function to be minimized comprises of cost of the device, line loadings and voltage deviations at the load buses.

\section{PROBLEM FORMULATION}

\section{A. Objective of the optimization}

As the cost of the FACTS devices is high, in order to achieve the maximum benefit, the devices are to be installed at the optimal locations. The objective function has three terms, the first term represents the installation cost of the devices, the second and third terms represent the load bus voltage deviations and line loadings respectively. The minimization of the proposed objective function has to lead to a cost effective security oriented device placement.

The objective function is formulated as

$\operatorname{Min} F=W_{1}\left[\left(C_{S V C} * S\right)+\left(C_{T C S C} * S\right)\right]+W_{2}[L V D]+W_{3}[L L]$

$F$ is the objective function;

$C_{T C S C}$ is the cost of TCSC device in US \$/KVar;

$C_{S V C}$ is the cost of SVC device in US \$/KVar;

$S$ is the operating range of the FACTS device;

$L V D$ is the Load voltage deviation;

$L L$ is the Line loading;

$W_{1}, W_{2} \& W_{3}$ are the weight factors.

(i) Cost $\left(C_{T C S C}\right) \&\left(C_{S V C}\right)$

The first term of the objective function $C_{T C S C}$ represents the installation cost of TCSC device and $C_{S V C}$ is the installation cost of SVC in the network, which are given by the following equations.

$$
\begin{aligned}
& C_{T C S C}=0.0015 s^{2}-0.7130 s+153.75 \\
& C_{S V C}=0.0003 s^{2}-0.3051 s+127.38
\end{aligned}
$$

(ii) Load voltage deviation $(L V D)$

Excessive high or low voltages can lead to an unacceptable service quality and can create voltage instability problems. FACTS devices connected at appropriate locations play a leading role in improving voltage profile thereby avoiding voltage collapse in the power system. The second term considered represents the load voltage deviations in order to prevent the under or over voltages at network buses.

$L V D=\sum_{m=1}^{n b}\left(\frac{V_{m r e f}-V_{m}}{V_{m r e f}}\right)^{n}$

$V_{m}$ is the voltage magnitude at bus $\mathrm{m}$

$V_{m r e f}$ is the nominal voltage at bus $\mathrm{m} \&$ is considered as $1.0 \mathrm{pu}$.

$m$ refers to the load buses, where $V_{m}$ is less than $V_{m r e f}$.

(iii) Line loading $(L L)$

TCSC is located in order to remove the overloads and to distribute the load flows uniformly. To achieve this, line loading is considered as the third term in the objective function. 
$L L=\sum_{l=1}^{n l}\left(\frac{S_{l}}{S_{l \max }}\right)^{n}$

$S_{l}$ is the apparent power in the line $l$.

$S_{l \text { max }}$ is the apparent power rating of line $l$.

\section{B. The optimization variables}

The optimization variables considered in this work are

(a) The number of FACTS devices (TCSC \& SVC) to be installed is taken as the first variable.

(b) The location of these devices is considered as the second variable to be optimized. TCSC is placed on a line and SVC is connected at a load bus. TCSC's are not installed in the lines where the transformers exist.

(c) Type of the device (TCSC or SVC) to be installed is considered as the third variable.

(d) The rating of the device is considered as the fourth variable.

Only one FACTS device per line or bus is permitted

\section{Modelling of FACTS devices}

\section{(i) TCSC Modelling}

TCSC is a series compensator. It consists of a series compensating capacitor shunted by a thyristor controlled reactor as shown in figure(a).With TCSC the power flow control can be done by increasing or decreasing the overall lines effective series transmission impedance, by adding a inductive or capacitive reactance correspondingly. The TCSC is modeled as a variable reactance as shown in figure (b).

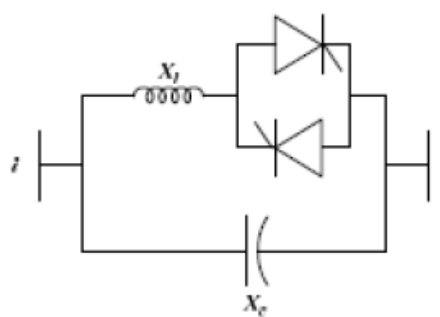

(a)

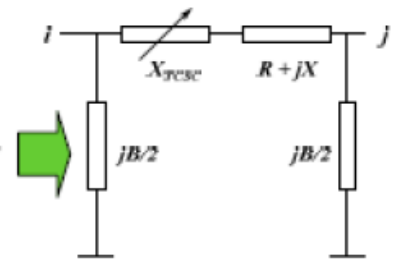

(b)

The working range of TCSC is considered as follows.

$-0.8 X_{l} \leq X_{T C S C} \leq 0.2 X_{l}$

$X_{T C S C}$ is the reactance added to the line by placing TCSC.

$X_{l}$ is the reactance of the line where TCSC is

located.

\section{(ii) SVC Modelling}

SVC is a shunt connected static var generator or absorber whose output is adjusted to exchange capacitive or inductive current so as to maintain or control specific parameters of the electrical power system. The SVC is a general term for a TCR (Thyristor Controlled Reactor), a TSC (Thyristor Switched Capacitor) or a combination of both as shown in figure (c). It works in two modes, capacitive or inductive mode. In inductive mode, it absorbs reactive power and in capacitive mode it injects reactive power. It is modeled as an ideal reactive power injection at bus $i$, where it is connected as depicted in figure (d). SVC is placed in only at load buses.

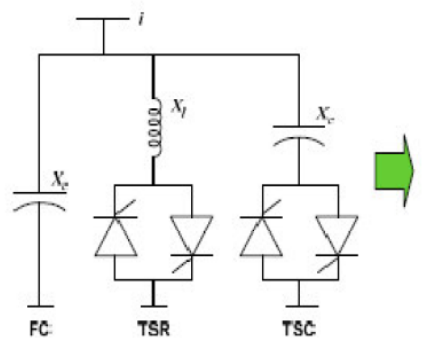

(c)

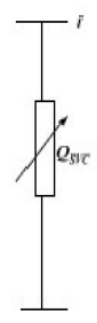

(d)

The reactive power is limited as follows

$Q_{S V C}^{\min } \leq Q_{S V C} \leq Q_{S V C}^{\max }$

Where

$Q_{S V C}^{\min }=-100 M V a r$

$Q_{S V C}^{\max }=100 M V a r$

III. OVERVEIW OF PSO TECHNIQUE 
Particle swarm optimization is a heuristic search technique developed by Eberhart and Kennedy [17] based on the concept of swarm intelligence exhibited by the flock of birds, school of fish etc in which each member of the group adjusts its behaviour based upon its own experience and the experience of the swarm. .This sort of social behaviour is used to simulate the problem solving environment in which a swarm is randomly generated in terms of solution variables of the problem. The individuals in a swarm are called particles. After generating the swarm, the fitness values of the particles $P_{\text {best }}$ are evaluated and compared against the values obtained from the previous iteration. The particles with the best values of fitness function in the next generation $P_{\text {best }}$ are retained. $G_{b e s t}$ is the best value attained so far by the swarm of particles. In each iteration, $G_{b e s t}$ of the current swam is compared with the $G_{b e s t}$ of the previous iteration and whichever is lower is retained along with the corresponding particle.

The position update of particles is carried out through the expression (8) in which the velocity is calculated using

$$
\begin{aligned}
& X_{i d}^{k+1}=X_{i d}^{k}+V_{i d}^{k+1} \\
& V_{i d}^{K+1}=W V_{i d}^{K}+c_{1} r_{1}\left(P_{b e s t i d}^{k}-X_{i d}^{k}\right)+c_{2} r_{2}\left(G_{b e s t i d}^{k}-X_{i d}^{k}\right)
\end{aligned}
$$

The inertia weight in (7) is calculated using the following expression.

$W=W_{\max }-\frac{W_{\max }-W_{\min }}{\text { iter }_{\max }} *$ iter

This iterative procedure is repeated till a specified number of swarms are reached or until a predefined amount of time has elapsed or until there is no considerable difference between the outcomes of a few subsequent iterations.

$V_{i d}^{k+1}$-Velocity of the $i^{t h}$ individual at $(k+1)^{t h}$ iteration.

$V_{i d}^{k}$-Velocity of the $i^{\text {th }}$ individual at $k^{\text {th }}$ iteration

$X_{i d}^{k}$ - Position of the $i^{\text {th }}$ individual at $k^{\text {th }}$ iteration.

$X_{i d}^{k+1}$ - Position of the $i^{\text {th }}$ individual at $(k+1)^{t h}$ iteration.

$P_{\text {best id }}$ - Best position of the $i^{\text {th }}$ individual.

$G_{\text {best id }}$ - Best position among the individuals.

$r_{1}, r_{2}$ - Random numbers distributed within the interval $[0,1]$

$c_{1}, c_{2}$ - Positive constants called acceleration constants.

$W$ - Inertia weight.

$W_{\max }$ - Initial value of inertia weight.

$W_{\min }$ - Final value of inertia weight.

iter $_{\text {max }}$ - Maximum number of iterations.

iter - Current iteration number.

$d=1,2, \ldots . D, D$ is the number of members in a particle.

$i=1,2, \ldots \ldots . m, m$ is the size of the swarm.

\section{ALGORITHM}

The algorithm of the proposed work is explained below.

Step1: The system data and the load factor are initialized.

Step2: PSO parameters such as the size of swarm m, maximum number of iterations, the number of variables to be optimized, limits of each variables in the particle, $c_{1} \& c_{2}$ values, $W_{\min } \& W_{\max }$, D, velocity limits, $P_{\text {best }}$ and $G_{b e s t}$ are initialized. Step3: An initial population is randomly generated considering the variables to be optimized. [The number of devices, location of the device, type of device, rating of the device]

Step4: For each particle $\mathrm{i}[\mathrm{i}=1,2 \ldots \mathrm{m}]$ in the population, the objective function is evaluated.

Stpe5: The calculated value of each particle is compared with its $P_{\text {best }}$ and $P_{\text {best }}$ of each particle is updated.

Step6: $G_{\text {best }}$ is calculated, then compared with the $G_{b e s t}$ in the previous iteration and it is updated.

Step7: A new population is created by changing the velocity and position of the particle. 
Step8: If stopping criterion is satisfied, the best individual is printed, else repeated from step 4. Step9: Steps from 2 to 8 are repeated for different load factors.

\section{SIMULATED RESULTS}

The proposed method has been tested on standard IEEE 14 bus, 30 bus and 57 bus test systems and the results are presented in tables 1 to 9. To study the effect of the installation of TCSC and SVC on load bus voltages and line loadings under overload conditions, the loads on the system were increased in a step by step manner; the real and reactive power loads connected at various load buses were increased keeping the load power factor constant. The maximum number of FACTS devices is limited to 2 on a 14 bus system, 3 on a 30 bus system and 5 on a 57 bus system. The test results are tabulated and an equivalent bar chart representation of the results is also presented for each of the load conditions.

The results are presented here for three different cases namely, variations of load bus voltages and line loadings under various loading conditions when no FACTS devices are present in the system, with only TCSC's connected to the system and with both TCSC as well as SVC connected.

Case (I) 14 bus system

Table1: Line loading for different load factors

\begin{tabular}{|l|c|c|c|c|c|c|}
\hline Load factor & 1 & 1.1 & 1.2 & 1.3 & 1.4 & 1.5 \\
\hline Without TCSC \& SVC & 17.5892 & 19.2093 & 20.9319 & 22.4464 & 24.0519 & 25.7766 \\
\hline With TCSC & 17.3907 & 19.0305 & 20.6862 & 22.3651 & 23.3228 & 25.0535 \\
\hline With TCSC \& SVC & 17.1084 & 18.8635 & 18.5792 & 20.5462 & 21.41 & 25.0779 \\
\hline
\end{tabular}

Table2: Load voltage deviation for different load factors

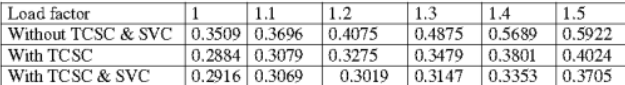

Table3: Location of FACTS devices

\begin{tabular}{|l|c|c|c|c|c|c|c|}
\hline & $\begin{array}{c}\text { Load } \\
\text { factor }\end{array}$ & 1 & 1.1 & 1.2 & 1.3 & 1.4 & 1.5 \\
\hline TCSC location & TCSC 1 & 12 & 12 & 4 & 12 & 6 & 12 \\
\hline Line number & TCSC 2 & 19 & 19 & 18 & 19 & 10 & 19 \\
\hline Line number & TCSC 1 & 18 & 12 & 18 & 18 & 18 & 5 \\
\hline TCSC \& SVC location \\
\hline Line number & SVC 1 & 13 & 13 & 13 & 10 & 13 & 8 \\
\hline Bus number &
\end{tabular}

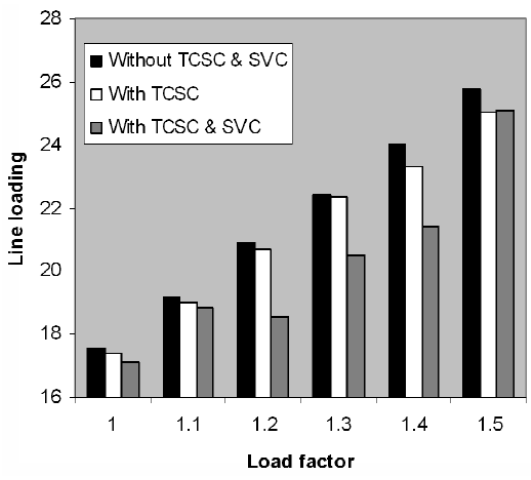

Fig. 1: Line loading VS percentage of load

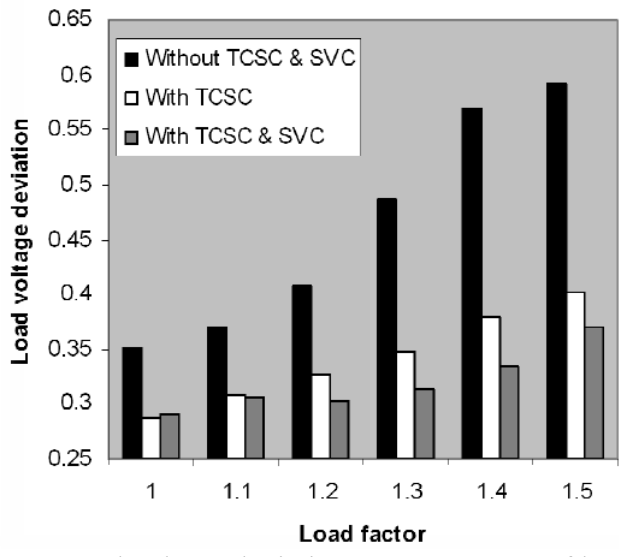

Fig. 2: Load voltage deviation VS percentage of load

\section{A. Case (II) 30 bus system}


Table4: Line loading for different load factors

\begin{tabular}{|l|c|c|c|c|c|c|}
\hline Load factor & 1 & 1.1 & 1.2 & 1.3 & 1.4 & 1.5 \\
\hline $\begin{array}{l}\text { Without TCSC \& } \\
\text { SVC }\end{array}$ & 14.5592 & 16.2116 & 17.9504 & 19.7258 & 21.5352 & 23.1761 \\
\hline With TCSC & 14.5522 & 16.1973 & 17.9397 & 19.7155 & 21.5319 & 23.1159 \\
\hline With TCSC \& SVC & 14.4592 & 16.1502 & 17.8509 & 19.5663 & 21.2701 & 23.0916 \\
\hline
\end{tabular}

Table6: Location of FACTS devices

\begin{tabular}{|l|c|c|c|c|c|c|c|}
\hline & $\begin{array}{c}\text { Load } \\
\text { factor }\end{array}$ & 1 & 1.1 & 1.2 & 1.3 & 1.4 & 1.5 \\
\hline TCSC location & TCSC 1 & 9 & 15 & 9 & 9 & 9 & 9 \\
\hline Line number & TCSC 2 & 25 & 28 & 25 & 21 & 21 & 21 \\
\hline Line number & TCSC 3 & 37 & 30 & 37 & 30 & 30 & 30 \\
\hline Line number & TCSC 1 & 37 & 10 & 39 & 9 & 37 & 9 \\
\hline TCSC \& SVC location \\
\hline Line number & SVC 1 & 13 & 13 & 13 & 13 & 13 & 13 \\
\hline Bus number & SVC 2 & 25 & 19 & 18 & 15 & 25 & 15 \\
\hline Bus number
\end{tabular}

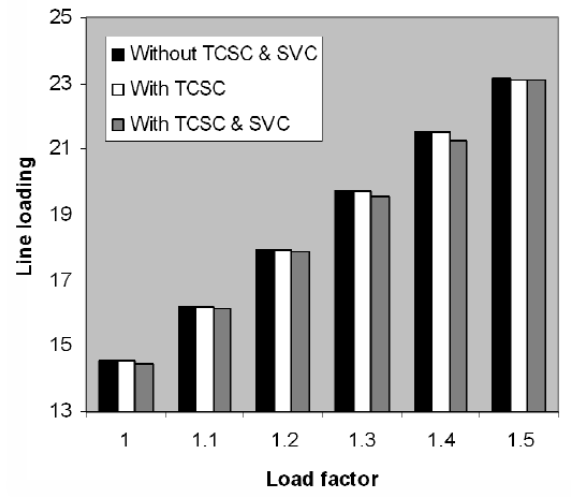

Fig. 3: Line loading VS percentage of load
Table5: Load voltage deviation for different load factors

\begin{tabular}{|l|c|c|c|c|c|c|}
\hline Load factor & 1 & 1.1 & 1.2 & 1.3 & 1.4 & 1.5 \\
\hline Without TCSC \& SVC & 0.6967 & 0.6974 & 0.7145 & 0.7342 & 0.7548 & 0.7834 \\
\hline With TCSC & 0.6965 & 0.6973 & 0.7128 & 0.7335 & 0.7529 & 0.7578 \\
\hline
\end{tabular} \begin{tabular}{|l|l|l|l|l|l|l|}
\hline With TCSC & 0.6965 & 0.6973 & 0.7128 & 0.7335 & 0.7529 & 0.7578 \\
\hline Wit TCSC SVC & 0.6952 & 0.6958 & 0.7103 & 0.7326 & 0.7515 & 0.7558 \\
\hline
\end{tabular} 


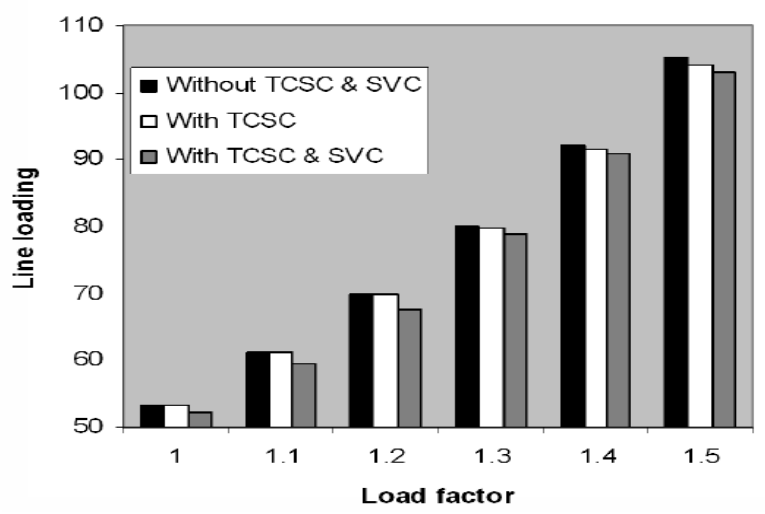

Fig.5: Line loading VS percentage of load

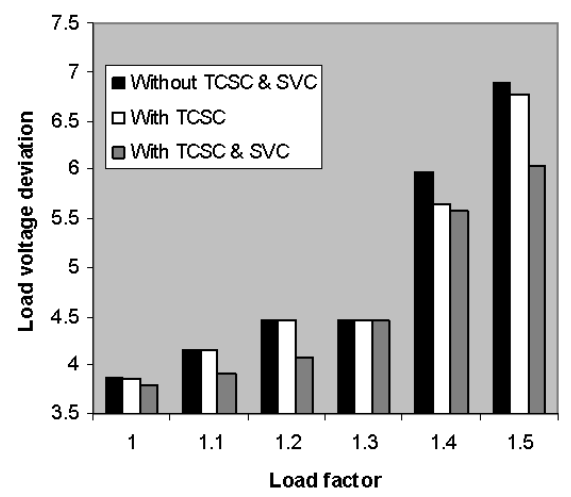

Fig. 6: Load voltage deviation VS percentage of load

It has been observed that for the IEEE 14 bus system, there is a significant reduction in the line loadings when TCSC's are connected at optimal locations. When SVC's are used along with TCSC's, line loadings are further reduced thereby improving the level of system security. It is also observed that the load voltage deviations are reduced when TCSC's are connected at appropriate locations and when SVC's were used along with TCSC's the load bus voltages improve significantly thereby resulting in a better voltage profiles throughout the system under normal and overload conditions. Similar results were obtained for IEEE 30 bus and 57 bus systems. The variations of line loadings and bus voltage deviations under various load conditions are presented in the form of bar charts in Figures 1 to 6 for better understanding.

\section{CONCLUSIONS}

A new method has been suggested for selecting the optimal location and size of TCSC's and SVC's under normal and overload conditions. The objective function formulated consists three terms, first term indicating the installation cost of the devices, the second term indicating the line loadings and the third one indicating the load voltage deviations. The objective function has been solved by the application of PSO technique which is basically a heuristic search algorithm which is well suited for complex, nonlinear problems. It has been found that the placement of TCSC's and SVC's of optimal ratings at suitable locations results in considerable improvement in bus voltage profiles and line loadings are reduced significantly.

\section{REFERENCES}

[1] Surekha Manoj, Dr. Puttaswamy P.S., "Importance of FACTS Controllers in power systems", International Journal of Advanced Engineering Technology, vol.II, Issue III, july-sep, 2011, pages 207-212.

[2] N.G.Hingorani and L.Gyugyi, "Understanding FACTS", IEEE Press, 1999.

[3] S.N.Singh, A.K.David, "Optimal location of FACTS devices for congestion management", Electric Power Systems Research vol 58, 2001, pages 71-79.

[4] R.Benabid, M.Boudour, M.A. Abido, "Optimal location and setting of SVC and TCSC devices using non-dominated sorting Particle Swarm Optimization”, Electric Power Systems Research vol 79, 2009, pages 1668-1677.

[5] K.Sundareswaran, P.Srinivasarao Nayak, Durga Venkatesh Ch, Hariharan B, "Optimal placement of FACTS devices using probabilistic Particle Swarm Optimization”, IEEE PES Innovative Smart Grid Technologies, 2011, pages1-6.

[6] Hugo Ambriz-Perez, Enrique Acha, Claudio R. Fuerte- Esquivel, "TCSC-firing angle model for optimal power flow solutions using Newton's method", Electric Power Systems Research vol 28, 2006, pages 77-85.

[7] Naresh Acharya, N.Mithulanathan, "locating series FACTS devices for congestion management in deregulated electricity markets", Electric Power Systems Research vol 77, 2007, pages 352-360.

[8] Ghamgeen I.Rashed, Yuanzhang Sun, Kai-Pei Liu, "Optimal placement of Thyristor Controlled Series Compensation in Power System based on Differential Evolution Algorithm" IEEE, seventh International Conference on Natural Computation, 2011, pages 2204-2210 
[9] S.Meikandasivam, Rajesh Kumar Nema, Shailendra Kumar Jain, "Behavioral Study of TCSC device- A matlab / simulink Implementation "World Academy of Science, Engineering and Technology, 2008. Pages 694-699.

[10] Preecha Preedavichit, S.C. Srivastava,"Optimal reactive power dispatch considering FACTS devices" Electric Power Systems Research vol 46, 1998, pages 251-257.

[11] K.Visaka, D.Thukaram, Lawrence Jenkins, "Application of UPFC for system security improvement under normal and network contingencies" electric power systems research-2003, pages 46-55.

[12] Sung-Hwan Song, Jung-Uk Lim, Seung-II Moon, "Installation and operation of FACTS devices for enhancing steady -state security" Electric Power Systems Research vol 70, 2004, pages 7-15.

[13] Stephane Gerbex, Rachid Cherkaoui, Alain J. Germond, "Optimal location of multi-type FACTS devices in a power system by means of genetic algorithms" IEEE Transactions on power systems, vol. 16. Aug. 2001, pages 537-544.

[14] Husam I.Shaheen, Ghamgeen I.Rashed, S.J. Cheng, "Optimal location and parameter setting of UPFC for enhancing power system security based on differential evolution algorithm", Electric Power Systems Research vol 33, 2010, pages 94-105.

[15] M.Saravanan, S.Mary Raja Slochanal, P.Venkatesh, J.Prince Stephen Abraham, "Application of particle swarm optimization technique for optimal location of FACTS devices considering cost of installation and system loadability", Electric Power Systems Research vol 77, 2007, pages 276-283.

[16] K.Satyanarayana, B.K.V.Prasad, G.Devanand, N.Siva Prasad, "optimal location of TCSC with minimum installation cost using PSO", International Journal of computer science and technology, vol 2, dec 2011, pages 156-160.

[17] James Kennedy, Russell Eberhart "Particle Swarm Optimization", in Proceedings of IEEE International Conference on Neural Networks, vol14, 1995, pages 1942-1948. 